\title{
Lifestyle intervention reduces CKD risk associated with type 2 diabetes
}

A secondary analysis of the Action for Health in Diabetes (Look AHEAD) randomized controlled trial has revealed that sustained weight loss might reduce the likelihood of very-high-risk chronic kidney disease $(\mathrm{CKD})$ in patients with type 2 diabetes mellitus (T2DM).

"Weight loss is part of standard treatment recommendations for overweight or obese persons with T2DM, but there is remarkably little evidence of its longterm value," explains lead author William Knowler of the NIH Institute of Diabetes and Digestive Kidney Diseases, AZ, USA. In the Look AHEAD trial, patients received either intensive lifestyle intervention (ILI), targeting a $\geq 7 \%$ decrease in body weight, or diabetes-specific support and education. Despite no significant differences in the primary study outcomes (death from cardiovascular causes or nonfatal myocardial infarction, stroke or hospitalization with angina), ILI resulted in a $31 \%$ reduction in risk of very-high-risk
CKD - as defined by KDIGO guidelinesafter a median follow-up duration of 8 years.

The researchers partly attribute this finding to significant reductions in body weight, blood glucose and systolic blood pressure in the ILI group, in which patients also used fewer antihypertensives.

Knowler concludes that "controlling blood pressure and glycaemia with medication is often not fully successful in preventing kidney disease in patients with T2DM. Lifestyle interventions aimed at weight loss should be considered as an additional intervention." He adds that longer term follow-up might reveal other significant differences in renal outcomes.

Peter Sidaway

Original article The Look AHEAD Research Group. Effect of a long-term behavioural weight loss intervention on nephropathy in overweight or obese adults with type 2 diabetes: a secondary analysis of the Look AHEAD randomised clinical trial. Lancet Diabetes Endocrinol. doi:10.1016/S2213-8587(14)70156-1 